\title{
DLC1 expression is reduced in human cutaneous melanoma and correlates with patient survival
}

\author{
Cecilia Sjoestroem ${ }^{1}$, Shahram Khosravi $^{1}$, Yabin Cheng ${ }^{1}$, Gholamreza Safaee Ardekani ${ }^{1}$, \\ Magdalena Martinka ${ }^{2}$ and Gang $\mathrm{Li}^{1}$ \\ ${ }^{1}$ Department of Dermatology and Skin Science, Vancouver Coastal Health Research Institute, University of \\ British Columbia, Vancouver, BC, Canada and ${ }^{2}$ Department of Pathology, Vancouver General Hospital, \\ Vancouver, BC, Canada
}

\begin{abstract}
Deleted in Liver Cancer-1 (DLC1) is a Rho-GTPase-activating protein known to be downregulated and function as a tumor suppressor in numerous solid and hematological cancers. Its expression status in melanoma is currently unknown however, prompting us to examine this. Using immunohistochemistry and tissue microarrays containing a large set of melanocytic lesions $(n=539)$, we examined the expression profile of DLC1 in melanoma progression, as well as the association between DLC1 and patient survival. We detected both cytoplasmic and nuclear DLC1 expression, and found that whereas cytoplasmic DLC1 was significantly downregulated in metastatic melanoma compared with nevi and primary melanoma, nuclear DLC1 expression was significantly down in primary melanoma compared with nevi, and then further down in metastatic melanoma. Loss of cytoplasmic DLC1 was significantly associated with poorer overall and disease-specific 5-year survival rates of all melanoma $(P<0.001$ and $P=0.001$, respectively) and metastatic melanoma patients $(P=0.020$ and 0.008 , respectively), and similar results were seen for nuclear DLC1 $(P<0.001$ for both overall and disease-specific survival for all melanoma patients, and $\boldsymbol{P}=\mathbf{0 . 0 0 4}$ for metastatic melanoma patients). Next, we examined the correlation between cytoplasmic and nuclear DLC1 and found that concomitant loss of both forms was associated with the worst outcome for metastatic melanoma patients $(P=0.013$ and $P=0.008$ for overall and disease-specific 5-year survival, respectively). Finally, multivariate Cox regression analysis determined that strong cytoplasmic and nuclear DLC1 expression was a favorable independent prognostic factor for all melanoma (HR, $0.61 ; 95 \% \mathrm{Cl}, 0.42-0.88 ; \boldsymbol{P}=0.008)$ and metastatic melanoma patients (HR, $0.42 ; 95 \%$ $\mathrm{Cl}, 0.23-0.77 ; \boldsymbol{P}=0.005)$. Although more research still needs to be done on the topic, these preliminary results support the hypothesis that DLC1 is a tumor suppressor in melanoma.

Modern Pathology (2014) 27, 1203-1211; doi:10.1038/modpathol.2013.223; published online 21 February 2014
\end{abstract}

Keywords: DLC1; immunohistochemistry; melanoma; metastasis; rhogap; tissue microarray; tumor suppressor

The incidence of cutaneous melanoma has been steadily increasing in the non-Hispanic White population worldwide since the $1950 \mathrm{~s}^{1}$ In the United States, incidence rates have increased by an average $2.8 \%$ per annum since 1992 in nonHispanic Whites, currently making it the fifth and seventh most commonly diagnosed cancer in men and women, respectively. ${ }^{2}$ Although melanoma

Correspondence: C Sjoestroem, MSc, Department of Dermatology and Skin Science, Vancouver Coastal Health Research Institute, University of British Columbia, Vancouver, BC, Canada.

E-mail: cecilia.sjoestroem@alumni.ubc.ca

Received 20 May 2013; revised 14 October 2013; accepted 15

October 2013; published online 21 February 2014 accounts for less than $5 \%$ of all skin cancers, it is responsible for over $80 \%$ of all skin cancer-related deaths. ${ }^{2}$ These numbers are greatly attributable to the high metastatic potential of melanoma, and are reflected by a 5 -year survival rate of only $5-16 \%$ for patients with distant metastases compared with a rate well above $90 \%$ for patients with early-stage, localized melanoma. ${ }^{2-5}$

Rho-GTPases belong to the small GTPase family of proteins, and are commonly implicated in the progression and metastasis of a number of cancers, including melanoma, where specifically RhoA, RhoC, and Cdc42 have all been positively associated with a metastatic phenotype. ${ }^{6,7}$ Rho-GTPase activity is dependent on the binding of either GTP or GDP 
and is controlled by three groups of proteins: GTPase-activating proteins (GAPs), guanine exchange factors (GEFs), and guanine nucleotide dissociation inhibitors. Whereas GEFs catalyze the nucleotide exchange, GAPs conversely catalyze the hydrolyzation of GTP to GDP, rendering the Rho-GTPases inactive. ${ }^{7-9}$

One RhoGAP protein extensively researched over the past decade is Deleted in Liver Cancer-1 (DLC1), a protein, as the name suggests, originally found to be deleted or downregulated in about $50 \%$ of primary hepatocellular carcinomas. ${ }^{10}$ DLC1 was mapped to chromosome $8 \mathrm{p} 21.3-22$, a region frequently deleted in many solid tumors, including liver cancer, but has later also been found to be silenced by other, mainly epigenetic, mechanisms. ${ }^{10-12}$ Since its initial discovery, several studies have reported DLC1 downregulation in a number of malignancies including breast, renal, lung, nasopharyngeal, esophageal, cervical, prostate, colorectal, oral squamous cell, and gastric carcinomas, as well as in multiple myelomas and lymphomas, ${ }^{13-23}$ and these studies together with recent in vitro and in vivo studies have confirmed the role of DLC1 as a bona fide tumor suppressor. $^{24-26}$

In addition to containing a RhoGAP domain, responsible for catalyzing the hydrolysis of GTP bound to RhoA, RhoB, RhoC, and Cdc42, DLC1 also contains a steroidogenic acute regulatoryrelated lipid transfer domain, a sterile alpha motif domain, and a focal adhesion-targeting (FAT) domain. ${ }^{27,28}$ The FAT domain is of particular interest as it has been shown to enhance the tumor suppressor activities of DLC1. ${ }^{29}$ It is well established that DLC1 is recruited to the focal adhesions via interactions between its FAT domain and the $\mathrm{SH} 2$ domains of the tensin proteins, which have moreover been found to differentially regulate DLC1 activity in vitro. ${ }^{30,31}$ Our group has recently found that one tensin family member, Cten, is upregulated in the progression from melanocytic nevi to primary tumors, ${ }^{32}$ and display oncogenic properties in vitro (unpublished data). This, along with the numerous studies showing DLC1 downregulation in cancer, prompted us to examine the expression profile of DLC1 in melanoma, as a first step to characterize its role in melanoma progression and metastasis.

Using immunohistochemical staining and tissue microarrays we examined the expression status of DLC1 in melanoma, as well as the correlation between DLC1 and patient survival. Our results show that DLC1 exists both as a cytoplasmic and a nuclear protein in melanocytic lesions, and indicate that downregulation of either cytoplasmic or nuclear DLC1 is associated with a significantly worse 5-year patient survival for metastatic melanoma patients, with concurrent loss of both being associated with the worst outcome.

\section{Materials and methods}

\section{Tissue Microarray Construction}

Seven hundred and forty-eight formalin-fixed, paraffin-embedded tissues were acquired from Vancouver General Hospital, Department of Pathology, between 1992 and 2009 in agreement with the Declaration of Helsinki guidelines, as approved by the Clinical Research Ethics Board of the University of British Columbia, Vancouver, BC, Canada. Tissues with lost cores or insufficient tumor cells were excluded from the study, leaving 539 tissues available for evaluation. The tissue microarrays were assembled as previously described. ${ }^{32,33}$

\section{Immunohistochemistry of Tissue Microarrays}

Deparaffinization of the tissue microarray slides was accomplished by heating the slides at $55^{\circ} \mathrm{C}$ for 20 min followed by three 5-min washes with xylene. Next, the slides were rehydrated by successive 5min washes in 100, 95 and $80 \%$ ethanol, and distilled water. Antigen retrieval was achieved by heating the samples at $95^{\circ} \mathrm{C}$ for $30 \mathrm{~min}$ in $10 \mathrm{mM}$ sodium citrate ( $\mathrm{pH} 6.0$ ), and endogenous peroxidase activity was blocked by incubation of the slides in $3 \%$ hydrogen peroxide for $30 \mathrm{~min}$. Next, the tissues were blocked with Dako antibody diluent (Dako Diagnostics, Glostrup, Denmark) for $30 \mathrm{~min}$, followed by incubation with a primary monoclonal mouse anti-DLC1 antibody (1:50 dilution, Santa Cruz Biotechnology, Santa Cruz, CA, USA) overnight at $4{ }^{\circ} \mathrm{C}$. The samples were then incubated with a universal biotinylated secondary antibody and streptavidin-HRP (Dako Diagnostics) for $30 \mathrm{~min}$ each, and developed using 3,3'-diaminobenzidine substrate (Vector Laboratories, Burlington, ON, Canada), followed by hematoxylin counterstaining.

\section{Evaluation of Immunostaining}

DLC1 staining was evaluated and scored based on intensity of staining (0-3) and percentage of DLC1expressing cells $(1(0-25 \%)$; $2(26-50 \%)$; 3 (51$75 \%)$; and $4(76-100 \%))$ by two independent observers. The degree of staining was calculated by multiplying the intensity score with the percentage of staining, and was accordingly classified as negative (0); weak (1-3); moderate (4-6); and strong (8-12). In the event of two duplicate cores having different staining, the higher of the two scores was used for the subsequent analysis.

\section{Statistical Analysis}

Differences in demographic and clinicopathological characteristics, and DLC1 expression between subgroups were evaluated by $\chi^{2}$-tests. Kaplan-Meier analyses and log-rank tests were used to evaluate the correlation between DLC1 expression and patient 
survival. Univariate and multivariate Cox regression analyses were used to determine the crude and adjusted hazard ratios, respectively, along with their 95\% confidence intervals. SPSS version 20.0 (SPSS, Chicago, IL, USA) software was used for all analyses, with a $P$-value $<0.05$ being considered significant.

\section{Results}

\section{Reduced DLC1 Expression Correlates with Melanoma} Progression and AJCC Stages

Tissue microarrays containing 34 normal nevi, 78 dysplastic nevi, 306 primary melanomas, and 121 metastatic melanomas were evaluated for DLC1 protein expression. DLC1 was expressed both in the cytoplasm and nuclei in melanocytic lesions (Figure 1), and both were further analyzed. Strong cytoplasmic DLC1 expression was detected in $65 \%$ of normal nevi,
$68 \%$ of dysplastic nevi, $61 \%$ of primary melanomas, and $50 \%$ of metastatic melanomas, with a significant decrease observed in metastatic melanomas compared with primary melanomas and dysplastic nevi $(P=$ 0.035 and $P=0.011$, respectively, $\chi^{2}$-test; Figure 1i). Strong nuclear DLC1 expression was observed in 82, 65 , 56 and $34 \%$ of normal nevi, dysplastic nevi, primary melanomas, and metastatic melanomas, respectively, with a significant difference seen between normal nevi and primary melanomas $(P=0.003)$, normal nevi and metastatic melanomas $(P<0.001)$, dysplastic nevi and metastatic melanomas $(P<0.001)$, and between primary and metastatic melanomas $(P<0.001)$. Furthermore, a borderline difference was seen between normal and dysplastic nevi $(P=0.070)$ and dysplastic nevi and primary melanomas $(P=$ 0.143 , $\chi^{2}$-test; Figure 1j). The correlation between cytoplasmic and nuclear DLC1 in melanoma progression is shown in Supplementary Figure S1.
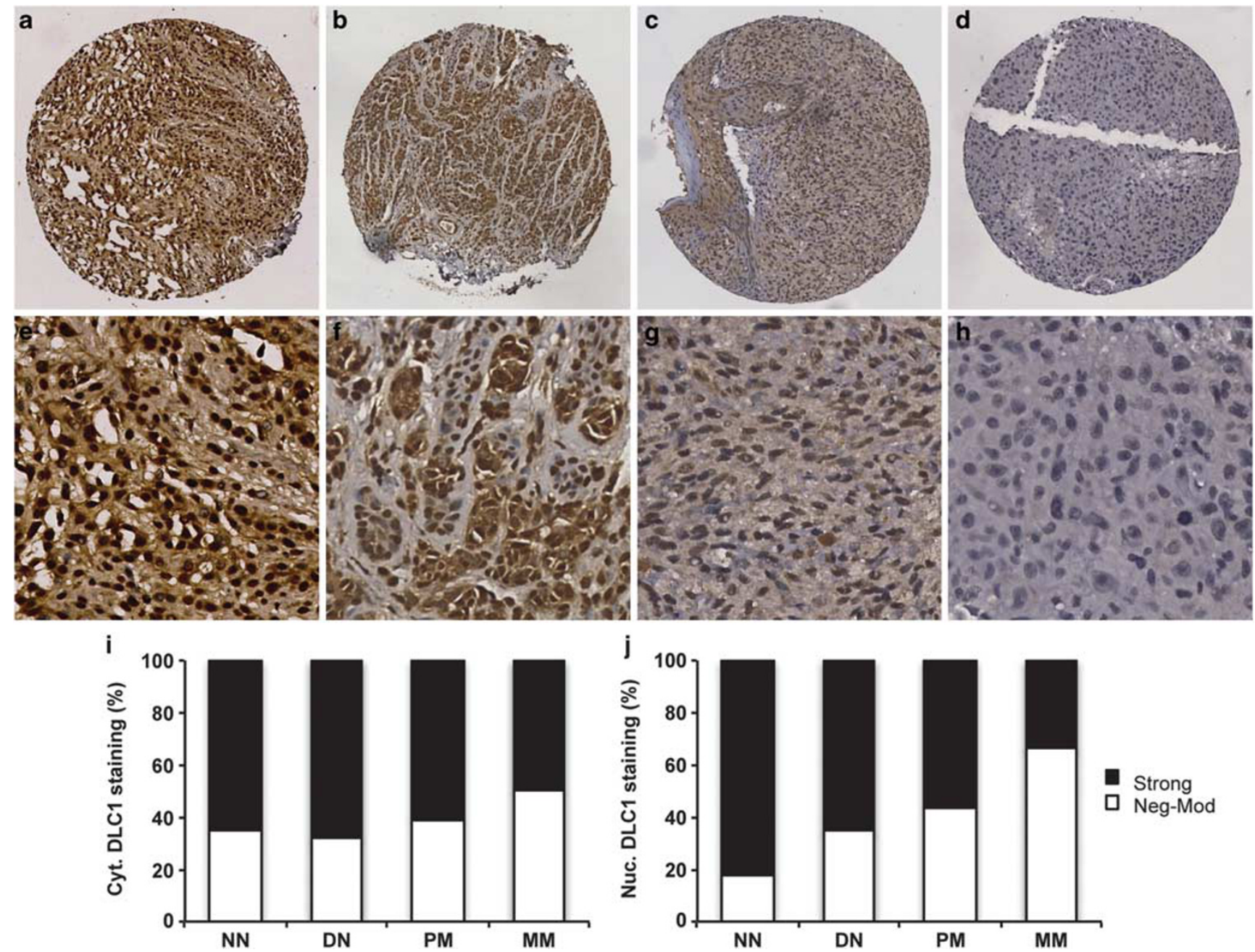

Figure 1 Representative images of cytoplasmic and nuclear DLC1 protein expression at $\times 100$ (a-d) and $\times 400$ magnification (e-h). (a, e) Strong cytoplasmic and nuclear DLC1 staining in normal nevi. (b, f) Strong cytoplasmic and moderate nuclear DLC1 staining in dysplastic nevi. (c, g) Moderate cytoplasmic and weak nuclear DLC1 staining in primary melanoma. (d, h) Weak cytoplasmic and negative nuclear DLC1 staining in metastatic melanoma. (i) Correlation between cytoplasmic DLC1 expression and melanoma progression. (j) Correlation between nuclear DLC1 expression and melanoma progression. DN, dysplastic nevi; MM, metastatic melanoma; NN, normal nevi; PM, primary melanoma. 
Next, the correlation between DLC1 and a number of demographic and clinicopathological characteristics was examined. One hundred and sixty-eight men and 137 women, with a median age of 59, were included in the analysis. Neither cytoplasmic nor nuclear DLC1 staining correlated with variables such as patient age and sex, primary tumor subtype, location, thickness, and status of ulceration (Supplementary Tables S1 and S2), but a significant association was found between DLC1 and AJCC stages (Figure 2). Strong cytoplasmic DLC1 expression was significantly reduced in tumors classified as AJCC stage IV (47\%), compared with AJCC stage I-III tumors (60\% strong staining, $P=0.046$, $\chi^{2}$-test; Figure 2a), whereas strong nuclear DLC1 staining was reduced in AJCC stage III + IV tumors $(35 \%)$ compared with AJCC stage I+II tumors (56\%, $P<0.001, \chi^{2}$-test; Figure 2b).

\section{DLC1 Protein Expression Correlates with Patient 5- and 10-Year Survival Rates}

A total of 396 melanoma patients (276 primary melanoma and 120 metastatic melanoma patients) had complete 5-year follow-up information, with survival times being calculated as the time from
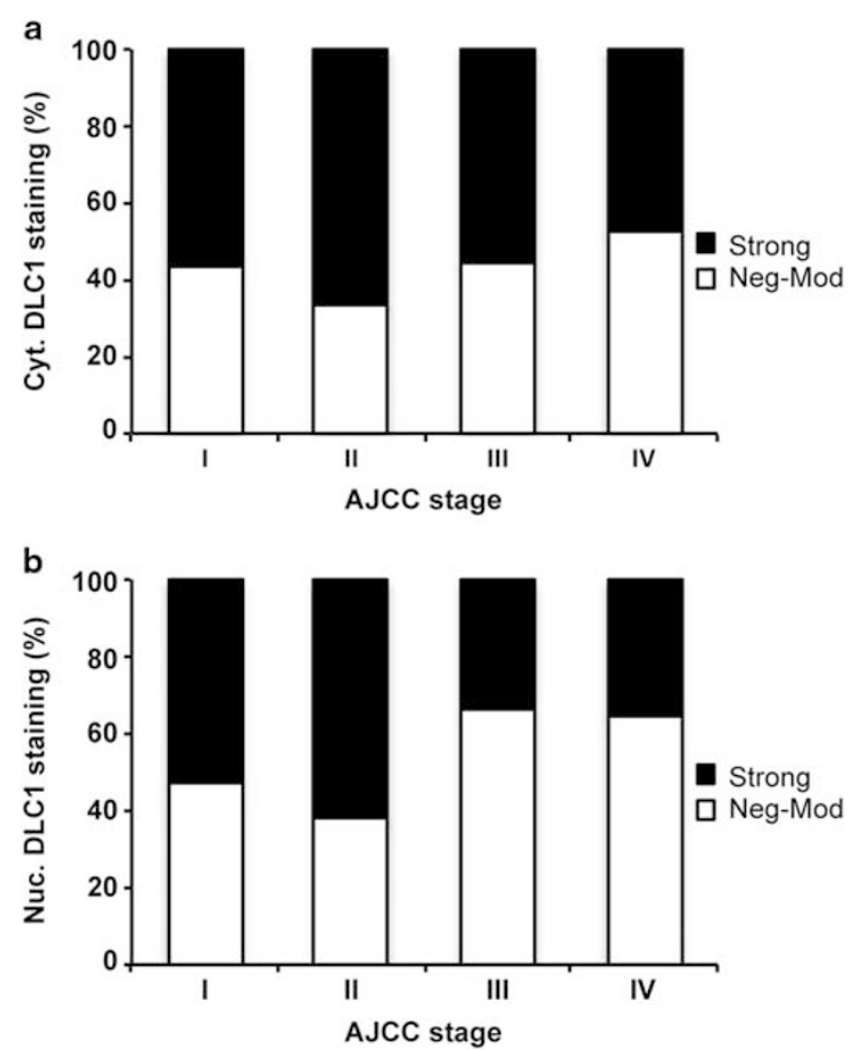

Figure 2 DLC1 expression correlates with AJCC stages. (a) Cytoplasmic DLC1 was significantly weaker expressed in AJCC stage IV tumors compared with AJCC stage I-III tumors $(P=0.046$, $\chi^{2}$-test). (b) Nuclear DLC1 was significantly weaker expressed in AJCC stage III + IV tumors, compared with AJCC stage I+II tumors $\left(P<0.001, \chi^{2}\right.$-test $)$. diagnosis to the last follow-up or death. To examine the correlation between DLC1 expression and patient survival, Kaplan-Meier survival curves were constructed. Analyses revealed that the expression level of both cytoplasmic and nuclear DLC1 was associated with the overall and disease-specific 5-year survival of all melanoma patients, with negative-moderate DLC1 expression being associated with a poorer survival outcome $(P<0.001$ and $P=0.001$ for cytoplasmic DLC1, and $P<0.001$ for both overall and disease-specific survival for nuclear DLC1; Figure 3). When divided into primary and metastatic melanoma patient groups, a significant association was seen between DLC1 and the overall and disease-specific survival of metastatic melanoma patients $(P=0.020$ and $P=0.008$ for cytoplasmic DLC1, and $P=0.004$ for both overall and disease-specific survival for nuclear DLC1; Figure 3) but not for primary melanoma patients (Supplementary Figure S2).

Both cytoplasmic and nuclear DLC1 were found to be significant risk factors for all melanoma and metastatic melanoma patients, as determined by univariate Cox regression analyses (Supplementary Table S3). Subsequent multivariate Cox regression analyses, adjusted to patient sex, age, and AJCC stage, showed that both cytoplasmic and nuclear DLC1, when analyzed separately, were independent prognostic factors for the 5-year survival of all melanoma and metastatic melanoma patients (Supplementary Tables S4 and S5).

Next, we were interested to see whether DLC1 was also associated with the overall and disease-specific 10 -year patient survival. Three hundred and twentynine patients had complete 10-year follow-up information. Whereas no association was found for primary or metastatic melanoma patients alone (data not shown); both cytoplasmic and nuclear DLC1 expression were significantly associated with the disease-specific 10-year survival of all melanoma patients $(P=0.046$ and $P=0.009$, respectively, Supplementary Figure S3).

\section{Concurrent Loss of Cytoplasmic and Nuclear DLC1 Expression is Associated with a Worse 5-Year Survival for Metastatic Melanoma Patients}

To examine the correlation between cytoplasmic and nuclear DLC1, and their effects on patient survival, the samples were divided into four groups based on their staining: (1) negative-moderate cytoplasmic and nuclear DLC1; (2) negative-moderate cytoplasmic DLC1 and strong nuclear DLC1; (3) strong cytoplasmic DLC1 and negative-moderate nuclear DLC1; and (4) strong cytoplasmic and nuclear DLC1. Figure 4 shows the results of Kaplan-Meier analyses. For all melanoma patients, loss of either or both cytoplasmic and nuclear DLC1 (categories 1-3) was associated with a poorer survival outcome compared with patients with strong cytoplasmic and nuclear DLC1 expression (category 4) in their tumors $(P<0.001$ for both overall and disease-specific 


\section{All melanoma patients}

Disease-specific survival
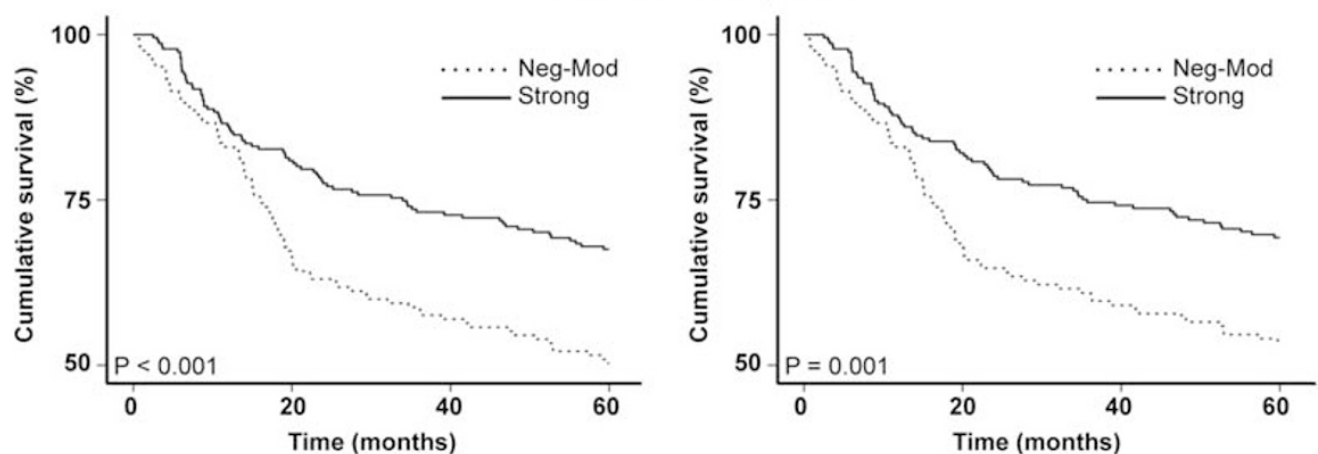

Metastatic melanoma patients
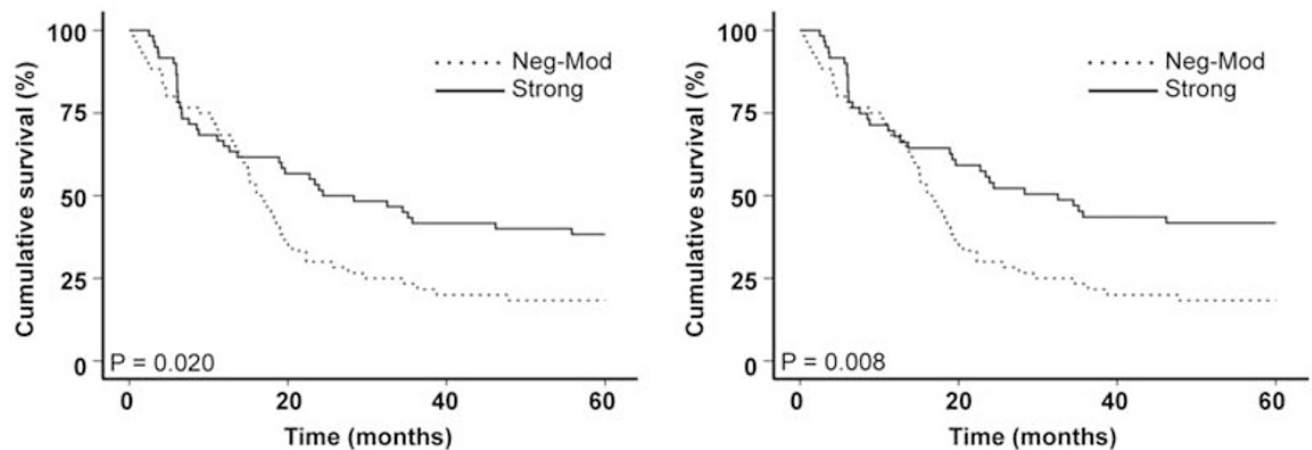

b

All melanoma patients
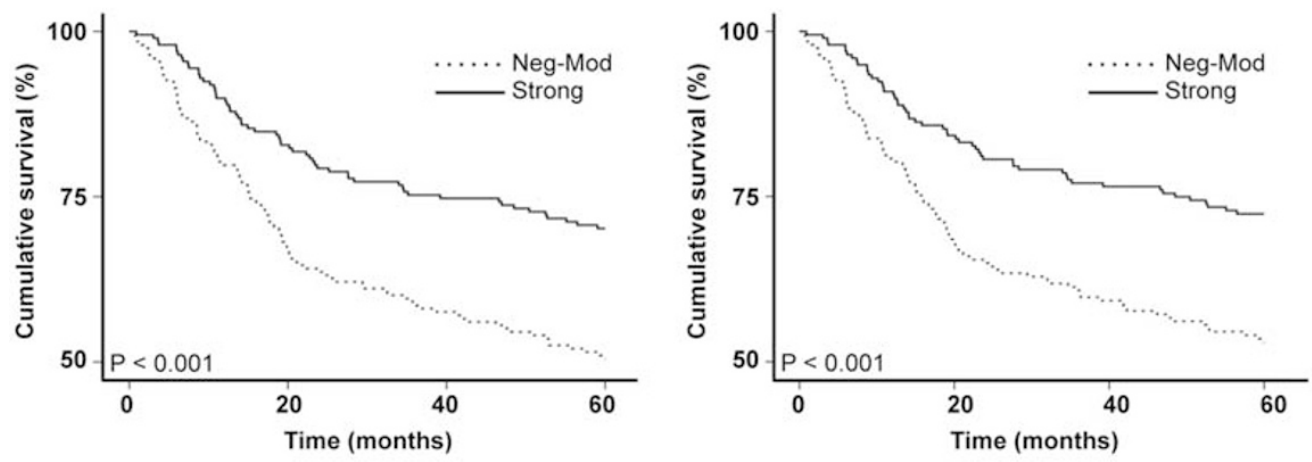

Metastatic melanoma patients
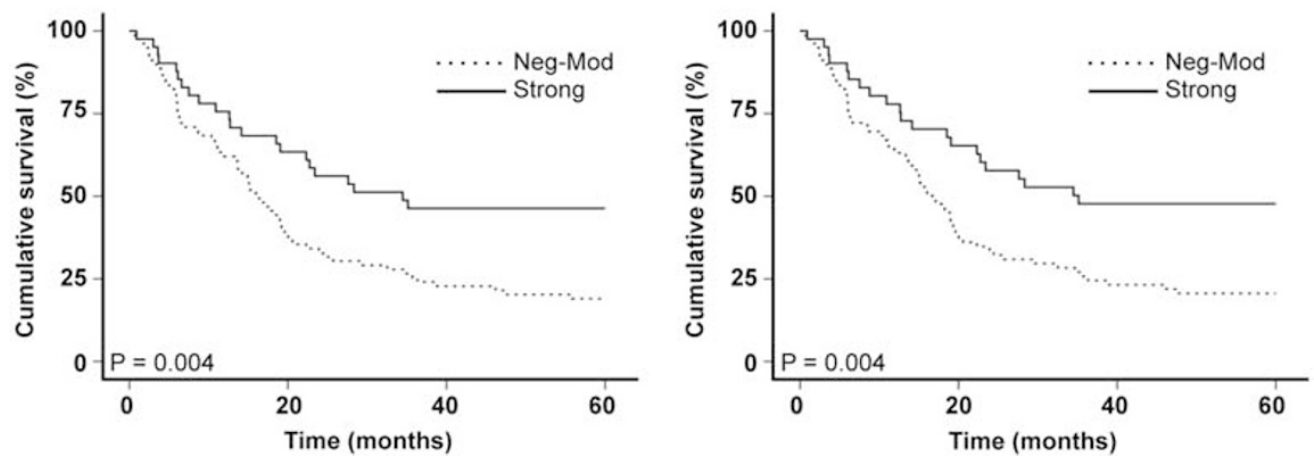

Figure 3 Kaplan-Meier analyses for the correlations between Deleted in Liver Cancer-1 (DLC1) expression and 5-year survival in melanoma patients. (a) Cytoplasmic DLC1 expression was significantly associated with the overall and disease-specific 5-year survival of all melanoma patients $(n=396, P<0.001$ and $P=0.001$, respectively, log-rank tests $)$ and metastatic melanoma patients $(n=120, P=0.020$ and $P=0.008$, respectively, log-rank tests). (b) Nuclear DLC1 expression was significantly associated with the overall and diseasespecific 5-year survival of all melanoma patients $(P<0.001$ for both, log-rank tests $)$ and metastatic melanoma patients $(P=0.004$ for both, log-ranks tests). 

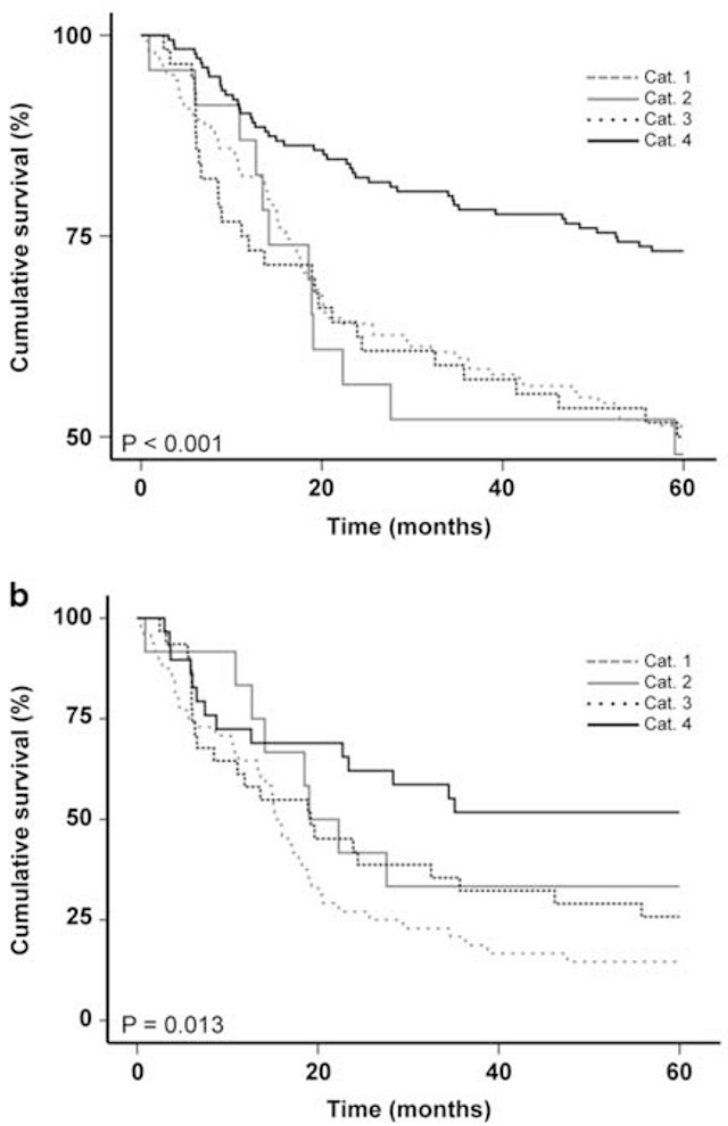

Disease-specific survival
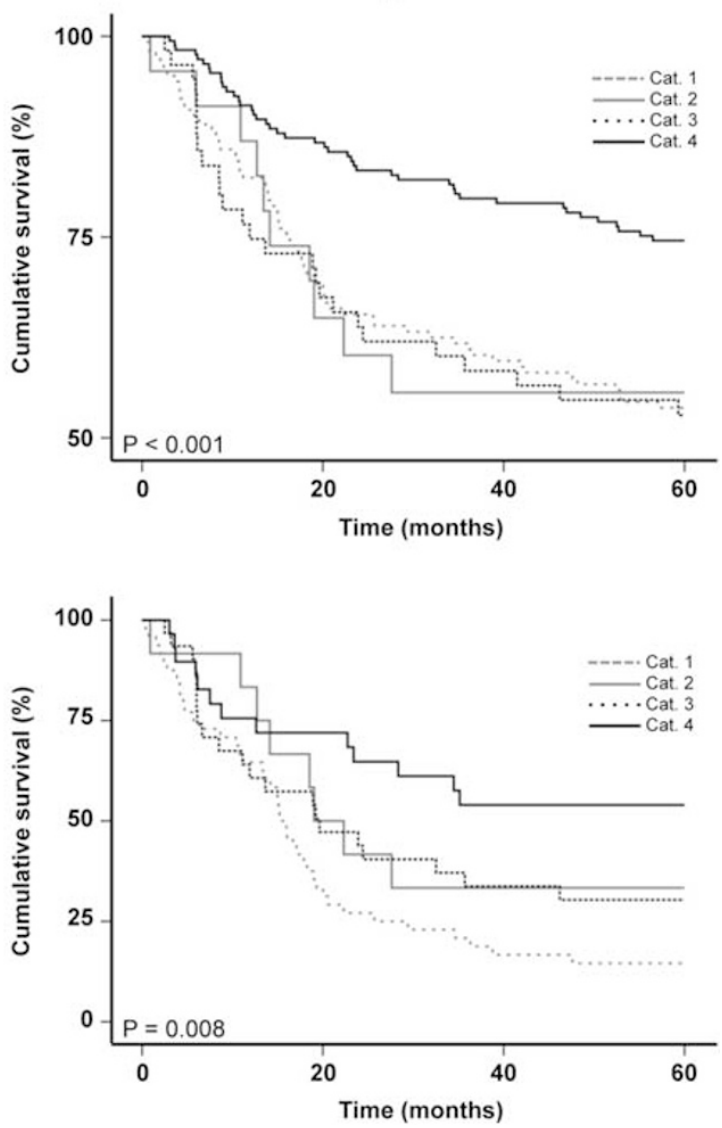

Figure 4 Simultaneous loss of cytoplasmic and nuclear Deleted in Liver Cancer-1 (DLC1) correlates with a poorer 5-year survival. (a) Strong cytoplasmic and strong nuclear DLC1 expression (category 4) was significantly associated with a better overall and diseasespecific 5-year survival outcome compared with loss of either or both cytoplasmic or nuclear DLC1 expression (category 1-3) in all melanoma patients $(P<0.001$, log-rank tests). (b) For metastatic melanoma patients only, strong cytoplasmic and nuclear DLC1 expression (category 4) was associated with the best 5-year survival outcome, whereas negative-moderate cytoplasmic and nuclear DLC1 expression (category 1) was associated with the worst outcome, and negative-moderate cytoplasmic and strong nuclear DLC1 (category 2) or strong cytoplasmic and negative-moderate nuclear DLC1 expression (category 3) were associated with an intermediate survival rate $(P=0.013$ and $P=0.008$ for overall and disease-specific survival, respectively, log-rank tests).

survival; Figure 4a). Interestingly, however, for metastatic melanoma patients, we found that while patients classified as category 4 still had the most favorable survival outcome (52 and $55 \%$ for overall and disease-specific 5-year survival, respectively), this time there was a clear difference between patients classified as categories 2 and 3 (33 and 26\% overall, and 33 and $32 \%$ disease-specific survival, respectively) and patients belonging to category $1(15 \%$ overall and disease-specific survival, $P=0.013$ and $P=0.008$, respectively; Figure $4 \mathrm{~b}$ ).

Multivariate Cox regression analysis further showed that strong expression of both cytoplasmic and nuclear DLC1 (category 4) was a favorable independent prognostic factor for all melanoma patients ((HR, 0.59; 95\% CI, 0.41-0.84; $P=0.003$ ) for overall survival and (HR, 0.61 ; 95\% CI, $0.42-$ $0.88 ; P=0.008)$ for disease-specific survival) and metastatic melanoma patients ((HR, 0.44; 95\% CI, $0.25-0.79 ; P=0.006$ ) for overall and (HR, 0.42; 95\%
CI, 0.23-0.77; $P=0.005$ ) for disease-specific survival, Table 1).

\section{Discussion}

In the present study, we examined the expression status of DLC1, a known tumor suppressor, in melanoma progression. To our knowledge, this is the first report on DLC1 downregulation in melanoma. Although the vast majority of publications regarding DLC1 have focused on its expression in the cytoplasm, we here discovered that DLC1 was expressed both in the cytoplasm and nuclei in human melanocytic lesions (Figure 1), and decided to investigate both further. Nuclear-localized DLC1, albeit less studied than its cytoplasmic counterpart, has recently been described in a handful of reports. In 2007, Yuan et $a l^{34}$ first reported that nuclear translocation of DLC1 preceded, and was required 
Table 1 Multivariate Cox regression analysis on 5-year overall and disease-specific survival of 396 melanoma patients

\begin{tabular}{|c|c|c|c|c|c|c|c|c|c|c|}
\hline \multirow[b]{2}{*}{ Variables $^{\mathrm{a}}$} & \multicolumn{5}{|c|}{ Overall survival } & \multicolumn{5}{|c|}{ Disease-specific survival } \\
\hline & $\beta^{\mathrm{b}}$ & $S E$ & $H R$ & $95 \% C I$ & P-value ${ }^{\mathrm{c}}$ & $\beta^{\mathrm{b}}$ & $S E$ & $H R$ & $95 \% C I$ & $\mathrm{P}$-value $\mathrm{c}$ \\
\hline \multicolumn{11}{|c|}{ All melanoma $(\mathrm{n}=396)$} \\
\hline Sex & 0.142 & 0.167 & 1.15 & $0.83-1.60$ & 0.393 & 0.167 & 0.173 & 1.18 & $0.84-1.66$ & 0.333 \\
\hline Age & 0.201 & 0.163 & 1.22 & $0.89-1.68$ & 0.217 & 0.226 & 0.169 & 1.25 & $0.90-1.75$ & 0.181 \\
\hline AJCC & 1.436 & 0.169 & 4.21 & $3.02-5.86$ & $<0.001$ & 1.522 & 0.176 & 4.58 & $3.25-6.47$ & $<0.001$ \\
\hline DLC1 & -0.529 & 0.180 & 0.59 & $0.41-0.84$ & 0.003 & -0.497 & 0.187 & 0.61 & $0.42-0.88$ & 0.008 \\
\hline \multicolumn{11}{|c|}{$M M(\mathrm{n}=120)$} \\
\hline Sex & 0.151 & 0.230 & 1.16 & $0.74-1.82$ & 0.511 & 0.208 & 0.232 & 1.23 & $0.78-1.94$ & 0.369 \\
\hline Age & 0.080 & 0.221 & 1.08 & $0.70-1.67$ & 0.718 & 0.107 & 0.225 & 1.11 & $0.72-1.73$ & 0.634 \\
\hline DLC1 & -0.816 & 0.298 & 0.44 & $0.25-0.79$ & 0.006 & -0.869 & 0.308 & 0.42 & $0.23-0.77$ & 0.005 \\
\hline
\end{tabular}

Abbreviations: CI, confidence interval; HR, hazard ratio; MM, metastatic melanoma.

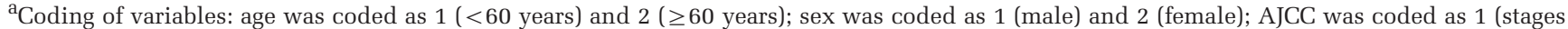
I + II) and 2 (stages III + IV); DLC1 was coded as 1 (categories 1, 2, and 3) and 2 (category 4).

$\mathrm{b} \beta=$ regression coefficient.

${ }^{\mathrm{C}}$ Log-rank test.

for, apoptosis in non-small cell lung carcinomas and that, similar to our results for normal nevi, nuclear DLC1 was highly expressed in human nonneoplastic alveolar epithelial cells. ${ }^{34}$ In 2008, Scholz et $a l^{35}$ showed that DLC1 was continuously shuttled between the cytoplasm and nuclei in cell lines, and yet another study from 2011 by Chan et $a l^{36}$ confirmed these results and further showed that DLC1 localized to the focal adhesions did not partake in the shuttling, and that nuclear DLC1 was less efficient in exerting its tumor suppressor activities compared with cytoplasmic DLC1. Whether or not this is also the case in melanoma remains to be investigated.

Supporting the proposed role of DLC1 as an inhibitor of cell migration and metastasis previously described in a number of cancers, ${ }^{22,23,27,31,37,38}$ we here found that DLC1 expression was reduced in metastatic melanoma compared with primary melanoma and nevi. Moreover, nuclear DLC1 expression was further down in primary melanomas compared with normal nevi, suggesting that loss of nuclear DLC1 may be an earlier event than cytoplasmic DLC1 loss in melanoma. This notion was further supported by the fact that cytoplasmic DLC1 expression was significantly lower in AJCC stage IV tumors compared with stage I-III tumors, whereas nuclear DLC1 expression was reduced in AJCC stage III tumors already. The importance of, and the mechanisms behind, this phenomenon will however need to be more closely examined before any conclusions about the interplay between cytoplasmic and nuclear DLC1 in melanoma can be drawn.

Both cytoplasmic and nuclear DLC1 were associated with the overall and disease-specific 5-year survival of all melanoma and metastatic melanoma patients (Figure 3), as well as with the diseasespecific 10-year survival of all melanoma patients
(Supplementary Figure S3). These results are consistent with the notion that DLC1 is a tumor suppressor in a variety of cancers. Interestingly, when we examined the correlation between cytoplasmic and nuclear DLC1, and their combined effects on patients' 5-year survival, we found that for metastatic melanoma patients, concurrent loss of both forms (category 1) was associated with the worst survival outcome, whereas loss of either cytoplasmic or nuclear DLC1 (categories 2 and 3) was associated with an intermediate survival outcome (Figure 4). This could imply that, similar to what has been reported in non-small cell lung carcinomas, ${ }^{34}$ the tumor suppressor properties of cytoplasmic and nuclear DLC1 differ from one another in melanoma. Thus, simultaneous loss of cytoplasmic and nuclear DLC1 could confer additive or synergistic effects on cancer progression, metastasis, and patient survival.

Finally, we used multivariate Cox regression analyses to investigate whether or not DLC1 is an independent prognostic factor for melanoma patients. Strong expression of cytoplasmic and nuclear DLC1 was found to be a favorable independent prognostic factor, both when analyzed separately (Supplementary Tables S4 and S5) and when analyzed together (Table 1), for the overall and disease-specific 5-year survival of all melanoma and metastatic melanoma patients. Currently, there is a lack of reliable prognostic markers used clinically for risk stratification, ${ }^{39}$ and although our results would have to be validated in a larger study first, they indicate that DLC1 has the potential to be a valuable prognostic marker for metastatic melanoma patients.

In conclusion, in this preliminary study we investigated the expression profile of DLC1 in melanoma and found that DLC1 was expressed both in the cytoplasm and nuclei at all stages of melanocytic lesions, and that both forms of DLC1 correlated with 
the 5-year survival of metastatic melanoma patients, with concurrent loss of both cytoplasmic and nuclear DLC1 being associated with the worst survival outcome. Although more work is still needed to elucidate the mechanisms behind the downregulation of DLC1 as well as the modes of action of both cytoplasmic and nuclear DLC1, these results support the notion that DLC1 is a tumor suppressor in melanoma.

\section{Acknowledgments}

We thank Scot Kwong for tissue microarray photography.

\section{Disclosure/conflict of interest}

The authors declare no conflict of interest.

\section{References}

1 Linos E, Swetter SM, Cockburn MG, et al. Increasing burden of melanoma in the United States. J Invest Dermatol 2009;129:1666-1674.

2 American Cancer Society Cancer Facts \& Figures 2011. American Cancer Society: Atlanta, 2011, p 60.

3 Gray-Schopfer V, Wellbrook C, Marais R. Melanoma biology and new targeted therapy. Nature 2007;445: 851-857.

4 Soengas MS, Lowe SW. Apoptosis and melanoma chemoresistance. Oncogene 2003;22:3138-3151.

5 Brown TJ, Nelson BR. Malignant melanoma: a clinical review. Cutis 1999;63:275-278.

6 Boone B, Van Gele M, Lambert J, et al. The role of RhoC in growth and metastatic capacity of melanoma. J Cutan Pathol 2009;36:629-636.

7 Gómez del Pulgar T, Benitah SA, Valerón PF, et al. Rho GTPase expression in tumourigenesis: evidence for a significant link. Bioessays 2005;27:602-613.

8 Vega FM, Ridley AJ. Rho GTPases in cancer cell biology. FEBS Lett 2008;582:1093-1101.

9 Ellenbroek SIJ, Gollard JG. Rho GTPases: functions and association with cancer. Clin Exp Metastasis 2007;24: 657-672.

10 Yuan BZ, Miller MJ, Keck CL, et al. Cloning, characterization, and chromosomal localization of a gene frequently deleted in human liver cancer (DLC-1) homologous to rat RhoGAP. Cancer Res 1998;58: 2196-2199.

11 Durkin ME, Yuan BZ, Zhou X, et al. DLC-1: a Rho GTPase-activating protein and tumour suppressor. J Cell Mol Med 2007;11:1185-1207.

12 Yuan BZ, Durkin ME, Popescu NC. Promoter hypermethylation of $D L C-1$, a candidate tumor suppressor gene, in several common human cancers. Cancer Genet Cytogenet 2003;140:113-117.

13 Yuan BZ, Zhou X, Durkin ME, et al. DLC-1 gene inhibits human breast cancer cell growth and in vivo tumorigenicity. Oncogene 2003;22:445-450.

14 Plaumann M, Seitz S, Frege R, et al. Analysis of DLC-1 expression in human breast cancer. J Cancer Res Clin Oncol 2003;129:349-354.
15 Zhang T, Zheng J, Liu C, et al. Expression of DLC-1 in clear cell renal carcinoma: prognostic significance for progression and metastasis. Urol Int 2009;82:380-387.

16 Yuan BZ, Jefferson AM, Baldwin KT, et al. DLC-1 operates as a tumor suppressor gene in human non-small cell lung carcinomas. Oncogene 2004;23: 1405-1411.

17 Seng TJ, Low JSW, Li H, et al. The major 8p22 tumor suppressor DLC1 is frequently silenced by methylation in both endemic and sporadic nasopharyngeal, esophageal, and cervical carcinomas, and inhibits tumor cell colony formation. Oncogene 2007;26:934-944.

18 Guan M, Zhou X, Soulitzis N, et al. Aberrant methylation and deacetylation of deleted in liver cancer-1 gene in prostate cancer: potential clinical applications. Clin Cancer Res 2006;12:1412-1419.

19 Peng H, Long F, Wu Z, et al. Downregulation of DLC-1 gene by promoter methylation during primary colorectal cancer progression. Biomed Red Int 2013;2013: 181384

20 Tripathi SC, Kaur J, Matta A, et al. Loss of DLC1 is an independent prognostic factor in patients with oral squamous cell carcinoma. Mod Pathol 2012;25:14-25.

$21 \mathrm{Kim}$ TY, Jong HS, Song SH, et al. Transcriptional silencing of the DLC-1 tumor suppressor gene by epigenetic mechanism in gastric cancer cells. Oncogene 2003;22:3943-3951.

22 Ullmannova-Benson V, Guan M, Zhou X, et al. DLC1 tumor suppressor gene inhibits migration and invasion of multiple myeloma cells through RhoA GTPase pathway. Leukemia 2009;23:383-390.

23 Ying J, Li H, Murray P, et al. Tumor-specific methylation of the 8p22 tumor suppressor gene DLC1 is an epigenetic biomarker for hodgkin, nasal NK/T-cell and other types of lymphomas. Epigenetics 2007;2:15-21.

24 Zhou X, Thorgeirsson SS, Popescu NC. Restoration of DLC-1 gene expression induces apoptosis and inhibits both cell growth and tumorigenicity in human hepatocellular carcinoma cells. Oncogene 2004;23: 1308-1313.

25 Xue W, Krasnitz A, Lucito R, et al. DLC1 is a chromosome 8p tumor suppressor whose loss promotes hepatocellular carcinoma. Genes Dev 2008;22:1439-1444.

26 Zhang $\mathrm{T}$, Zheng J, Jiang N, et al. Overexpression of DLC-1 induces cell apoptosis and proliferation inhibition in the renal cell carcinoma. Cancer Lett 2009;83: 59-67.

27 Healy KD, Hodgson L, Kim TY, et al. DLC-1 suppresses non-small cell lung cancer growth and invasion by RhoGAP-dependent and independent mechanisms. Mol Carcinog 2008;47:326-337.

28 Liao YC, Lo SH. Deleted in liver cancer-1 (DLC-1): a tumor suppressor not just for liver. Int J Biochem Cell Biol 2008;40:843-847.

29 Liao YC, Shih YP, Lo SH. Mutations in the focal adhesion targeting region of deleted in liver cancer-1 attenuate their expression and function. Cancer Res 2006;68:7718-7722.

30 Liao YC, Si L, DeVere White RW, et al. The phosphotyrosine-independent interaction of DLC-1 and the SH2 domain of cten regulates focal adhesion localization and growth suppression activity of DLC-1. J Cell Biol 2007;176:43-49.

31 Cao X, Voss C, Zhao B, et al. Li SSC. Differential regulation of the activity of deleted in liver cancer 1 (DLC1) by tensins controls cell migration and transformation. Proc Natl Acad Sci USA 2012;109:1455-1460. 
32 Sjoestroem C, Khosravi S, Zhang G, et al. C-terminal tensin-like protein is a novel prognostic marker for primary melanoma patients. PLoS ONE 2013;8:e80492.

33 Dai DL, Martinka M, Li G. Prognostic significance of activated akt expression in melanoma: a clinicopathologic study of 292 cases. J Clin Onc 2005;7:1473-1482.

34 Yuan BZ, Jefferson AM, Millecchia L, et al. Morphological changes and nuclear translocation of DLC1 tumor suppressor protein precede apoptosis in human non-small cell lung carcinoma cells. Exp Cell Res 2007;313:3868-3880.

35 Scholz RP, Regner J, Theil A, et al. DLC1 interacts with 14-3-3 proteins to inhibit RhoGAP activity and block nucleocytoplasmic shuttling. J Cell Sci 2008;122: 92-102.
36 Chan LK, Ko FCF, Sze KMF, et al. Nuclear-targeted deleted in liver cancer 1 (DLC1) is less efficient in exerting its tumor suppressive activity both in vitro and in vivo. PLoS ONE 2011;6:e25547.

37 Chen WT, Yang CH, Wu CC, et al. Aberrant deleted in liver cancer-1 expression is associated with tumor metastasis and poor prognosis in urothelial carcinoma. Acta Path Micro Im 2013;121:1131-1138.

38 Goodison S, Yuan J, Sloan D, et al. The RhoGAP protein DLC-1 functions as a metastasis suppressor in breast cancer cells. Cancer Res 2005;65:6042-6053.

39 Rothberg BE, Bracken MB, Rimm DL. Tissue biomarkers for prognosis in cutaneous melanoma: a systematic review and meta-analysis. J Natl Cancer Inst 2009;101:452-474.

Supplementary Information accompanies the paper on Modern Pathology website (http://www.nature.com/ modpathol) 